\title{
Exciton Dynamics of Colloidal Semiconductor Quantum Well Stacks
}

\author{
Onur Erdem, Burak Guzelturk, Murat Olutas, Yusuf Kelestemur, \\ and Hilmi Volkan Demir
}

\begin{abstract}
Colloidal semiconductor nanoplatelets (NPLs) have recently emerged as a new class of colloidal nanocrystals. NPLs are quasi two-dimensional nanocrystals having atomically flat surfaces and have unique properties such as narrow photoluminescence $(\mathrm{PL})$ emission $(\sim 10 \mathrm{~nm})$ and giant oscillator strength. NPLs can be self-assembled into stacks. These are one-dimensional superstructures that can contain tens or hundreds of NPLs in one chain.

We studied how stacking modifies the optical properties of NPLs.

We found that PL quantum yield and exciton lifetime are reduced with increased degree of stacking in NPL ensembles. Moreover, we showed that temperaturedependent behavior of stacked NPLs is significantly different than the nonstacked ones. We developed two statistical models that account for the ultra-fast nonradiative energy transfer within stacked NPL chains as well as nonemissive subpopulation of NPLs in the ensemble to explain the aforementioned changes when NPLs are stacked.
\end{abstract}

Colloidal nanoplatelets (NPLs) are solution-processed quantum wells with magic sized vertical thickness of few nm's and lateral sizes of 10-100 nm. Since the lateral sizes are typically larger than the exciton Bohr radius, the quantum confinement in these nanostructures is strong only in the vertical direction. This leads to unique

O. Erdem $\cdot$ B. Guzelturk $\cdot$ M. Olutas $\cdot$ Y. Kelestemur

Department of Electrical and Electronics Engineering, Department of Physics, UNAM - Institute of Materials Science and Nanotechnology, Bilkent University, Ankara, Turkey

H. V. Demir $(\square)$

Department of Electrical and Electronics Engineering, Department of Physics, UNAM - Institute of Materials Science and Nanotechnology, Bilkent University, Ankara, Turkey

Luminous! Center of Excellence for Semiconductor Lighting and Displays, School of Electrical and Electronic Engineering, School of Physical and Materials Sciences, School of Materials Science and Nanotechnology, Nanyang Technological University, Singapore, Singapore e-mail: volkan@fen.bilkent.edu.tr

B. Di Bartolo et al. (eds.), Quantum Nano-Photonics, NATO Science for Peace and Security Series B: Physics and Biophysics, https://doi.org/10.1007/978-94-024-1544-5_20 

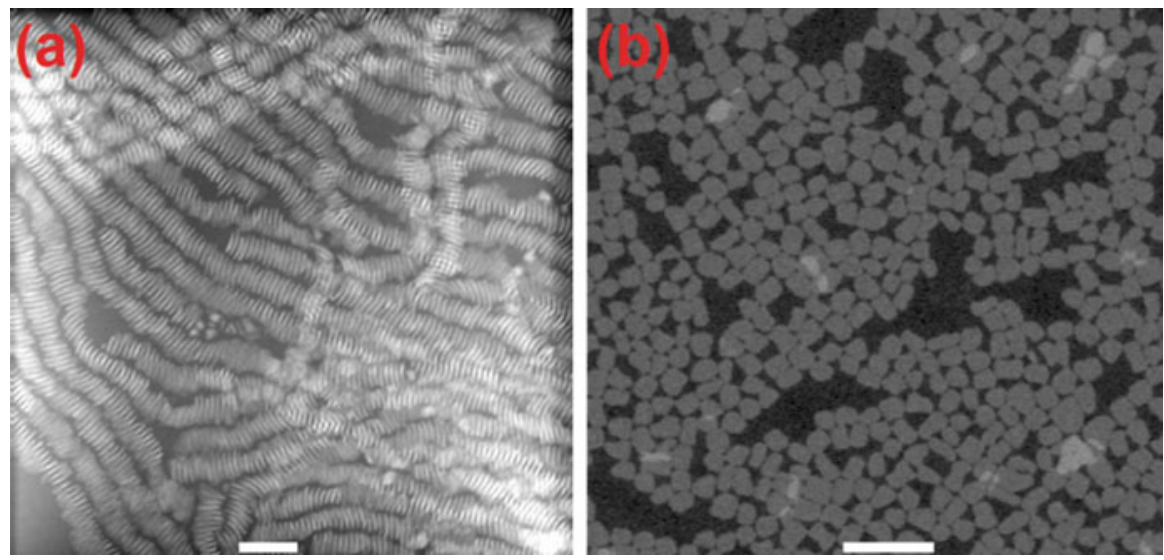

Fig. 1 Transmission electron microscopy images of (a) stacked and (b) nonstacked nanoplatelets. Scale bars are $50 \mathrm{~nm}$ (Demir Group)

properties in the NPLs including narrow photoluminescence (PL) emission $(<$ $10 \mathrm{~nm}$ ) and giant oscillator strength transition.

NPLs can assemble themselves into one-dimensional long chains, which are commonly referred to as stacks (Fig. 1a). When stacked, the NPLs are aligned face to face, standing on their edges instead of lying flat on the substrate (Fig. 1b). We observed that stacking strongly modifies the optical properties of the NPLs. For instance, the PL lifetime and quantum yield (QY) of the NPLs and the are reduced with increasing degree of stacking [1]. Moreover, the increase in QY with decreasing temperature is suppressed when the NPLs are in stacked formation [2].

To account for these modifications we developed two mathematical models making use of the fact that there is ultra-fast exciton transfer between neighboring NPLs of a stack, which leads to increased hole trapping in defected NPLs. The first one constructs and solves a set of rate equations to determine the lifetime of the exciton decay [1]. The second approach treats the movement of the excitation between the NPLs as a random walk and utilizes Markov chains to estimate the exciton lifetime and QY of the stacks. Using experimental data, the rates of energy transfer and exciton recombination are calculated and used in the construction of state transition matrices [2]. Using this model, we are able to calculate the changes in QY and PL lifetimes with temperature. Both models show good agreement with the experimental data. 


\section{References}

1. Guzelturk B, Erdem O, Olutas M, Kelestemur Y, Demir HV (2014) Stacking in colloidal nanoplatelets: tuning excitonic properties. ACS Nano 8(12):12524-12533

2. Erdem O, Oluras M, Guzelturk B, Kelestemur Y, Demir HV (2016) Temperature-dependent emission kinetics of colloidal nanoplatelets strongly modified by stacking. J Phys Chem Lett 7(3):548-554 